\title{
Stent Direto em Tronco Protegido de Artéria Coronária Esquerda
}

\author{
Maria da Conceição Alves Pinto' ${ }^{1}$, Nahaliel Pessoa Rodrigues ${ }^{1}$
}

\section{RESUMO}

Paciente de 82 anos, masculino, portador de doença arterial coronariana (DAC), cirurgia de revascularização miocárdica (CRVM) em 1986, Angioplastia transluminal percutânea (PTCA) com stent na anastomose distal da Ponte de Artéria torácica interna esquerda (ATIE)/Artéria Descendente Anterior (ACDA) em 2003, e atualmente com angina instável de alto risco. Coronariografia mostra lesão obstrutiva grave na porção distal do Tronco de Artéria Coronária Esquerda (TCE), Pontes de safenas ocluídas (PS) e ponte da ATIE anastomosada com ACDA patente. O referido paciente foi submetido a angioplastia coronariana de TCE protegido/ artéria circunflexa (ACX) com implante de stent com eluição de paclitaxel, com sucesso.

DESCRITORES: Angina instável. Angioplastia transluminal percutânea coronária. Contenedores. Paclitaxel.
A Cirurgia de Revascularização Miocárdica é ainda considerada o tratamento de escolha para os pacientes com estenose significativa em Tronco de Artéria Coronária Esquerda (TCE), principalmente nos casos de maior complexidade anatômica ${ }^{1}$, limitando, assim, as recomendações das intervenções percutâneas em lesões de TCE com stent, reservando apenas para os subgrupos de pacientes com risco cirúrgico inaceitável. Contudo, de acordo com as diretrizes atuais da ACC/ AHA/ SCAI 2005 - Guideline Update for Percutaneous Coronary Intervention ${ }^{2}$, em pacientes que foram submetidos a cirurgia de revascularização miocárdica, a intervenção percutânea coronariana pode ser realizada em outros vasos com obstruções significativas, quando tecnicamente viável e desde que haja patência da ponte da artéria torácica interna (Recomendação classe Ila). Baseado também nesta diretriz ${ }^{2}$, se o clopi-

\footnotetext{
${ }^{1}$ Hospital Adventista Silvestre - Santa Teresa - RJ - Brasil.

Correspondência: Maria da Conceição Alves Pinto. Hospital Adventista Silvestre/Serviço de Hemodinâmica. Ladeira dos Guararapes, 263 Santa Teresa - RJ - CEP 22241-220

E-mail: mariaceicao@hotmail.com

Recebido em: 6/2/2007 - Aceito em: 7/5/2007
}

\begin{abstract}
SUMMARY
Direct Stenting of a Protected Left

Coronary Artery Trunk

This report describes the case of an 82-year-old male patient with coronary artery disease; he underwent CABG in 1986, $\mathrm{PCl}$ with bare stent placement in the distal anastomosis of a left internal thoracic artery (LITA)/anterior descending artery (ADA) in 2003 and at the time of this report was at high risk for unstable angina. Coronary angiography demostrated severe stenosis of the $1 / 3$ of the left coronary artery trunk, saphenous vein grafts and the LITA graft anastomosed to the anterior descending artery patent. The patient was successfully treated by coronary angioplasty of the "protected" left coronary artery trunk/ circumflex artery using a paclitaxel eluting stent.
\end{abstract}

DESCRIPTORS: Angina, unstable. Angioplasty, transluminal, percutaneous coronary. Stents. Paclitaxel.

dogrel é administrado no momento da intervenção, a complementação com os inibidores da glicoproteína IIb/ Illa pode ser benéfica para facilitar precocemente a inibição plaquetária, especialmente entre os pacientes de alto risco (Recomendação classe Ila). Em relação ao emprego de stents com eluição de fármacos antiproliferativos (sirolimus, paclitaxel), nestes mesmos pacientes, os estudos ${ }^{3-8}$ mostraram resultados mais promissores, quando comparados ao stents convencionais.

\section{RELATO DO CASO}

Paciente do sexo masculino, 82 anos, hipertenso, dislipidêmico, submetido a cirurgia de revascularização miocárdica, em 1986 (ATIE/ACDA, PS-AO/ACD, PSAO/MG). Assintomático até 2003, quando iniciou quadro de angina de esforço, sendo submetido a teste ergométrico com critérios positivos para isquemia miocárdica. Coronariografia na época mostrava: tronco coronariano esquerdo com lesão obstrutiva (60\%) na porção distal, comprometendo a origem da artéria coronária descendente anterior e a circunflexa. Artéria coronária descendente anterior e coronária direita ocluídas no $1 / 3$ proximal, sendo que esta opacifica seu leito distal por colaterais. Pontes de safenas pérvias e 
Pinto MCA e Rodrigues NP. Stent Direto em Tronco Protegido de Artéria Coronária Esquerda. Rev Bras Cardiol Invas 2007; 15(3): 307-310.

lesão obstrutiva grave na anastomose distal da ponte da ATIE/ACDA, submetido a angioplastia transluminal percutânea (PTCA) com stent convencional, com sucesso. Internado, em janeiro de 2007, com quadro de angina instável de alto risco (TIMI risco 6). Realizado estudo coronariográfico que mostrava: lesão obstrutiva grave $(90 \%)$ na porção distal do tronco coronariano esquerdo, comprometendo a origem da artéria circunflexa e da descendente anterior, a qual se mostrava ocluída no 1/3 proximal; pontes de safenas e artéria coronária direita ocluídas, ponte da ATIE/ACDA patente, com stent pérvio; ventriculografia esquerda com acinesia inferior, valva mitral competente (Figuras 1 a 7). Em virtude da gravidade do quadro clínico, da necessidade de se intervir precocemente e por não haver uma antiagregação plaquetária prévia satisfatória, iniciamos inibidor da glicoproteína Ilb/lla (Abciximab)+300mg de clopidogrel+200mg de ácido acetilsalicílico na sala de hemodinâmica. Imediatamente, posicionamos o cate-

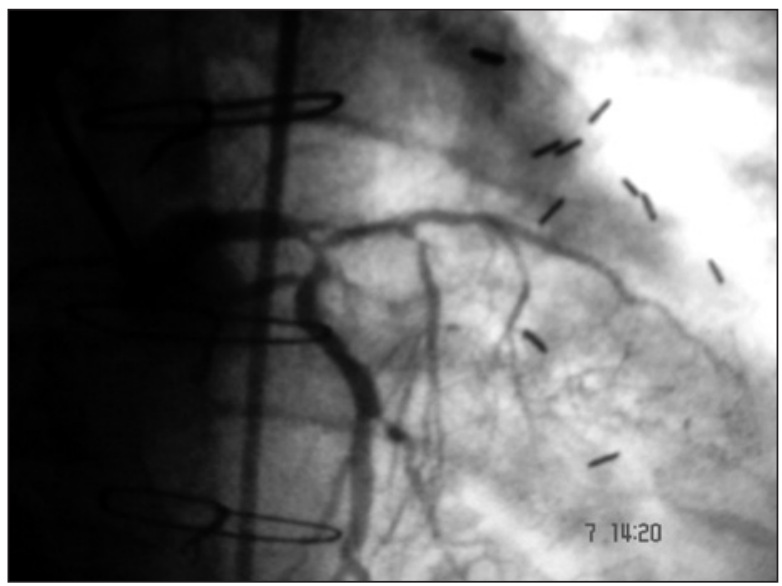

Figura 1 - Coronariografia esquerda em projeção PA. A artéria coronária esquerda apresenta lesão obstrutiva grave na porção distal do tronco coronariano esquerdo.

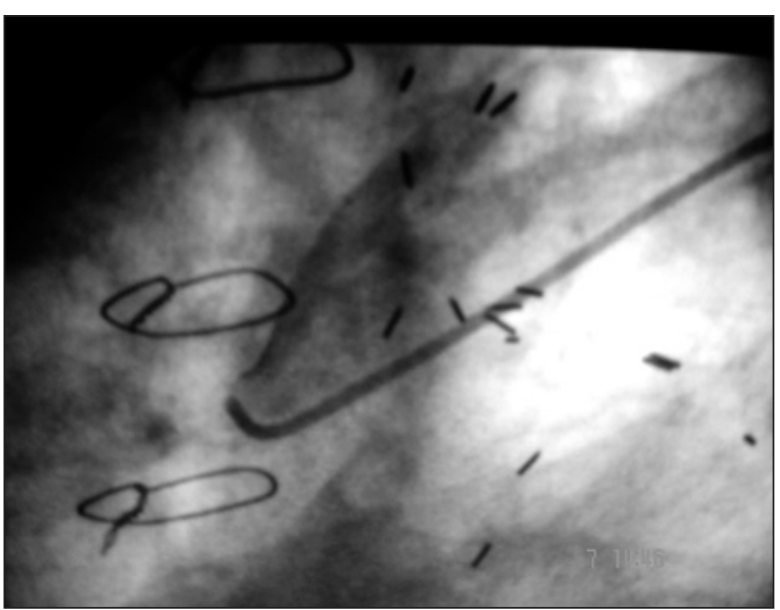

Figura 2 - Estudo da ponte de safena em projeção $O A E$, onde se observa o nicho da ponte de safena. ter-guia EBU - 6F e, após injeção de contraste, visualizamos lesão grave na porção distal do tronco coronariano esquerdo e origem da artéria circunflexa, foi passada a corda-guia 0,014 e, em seguida, implantado o stent TAXUS - Liberté $(3,0 \times 16)$ a 16atm, pela técnica "direct stent", com sucesso angiográfico (Figuras 8 a 10). A alta hospitalar ocorreu 72 horas após, estando o paciente assintomático. Orientado a fazer uso de clopidogrel por, no mínimo, um ano, uso contínuo da aspirina e estatina, além do controle rigoroso dos fatores de risco para doença arterial coronariana.

\section{DISCUSSÃO}

O caso relatado se enquadra nas diretrizes acima descritas, por ser um paciente com revascularização miocárdica prévia, conduto arterial pérvio e risco elevado. Em relação à artéria coronária direita, resolvemos não intervir, pois esta já se encontrava ocluída

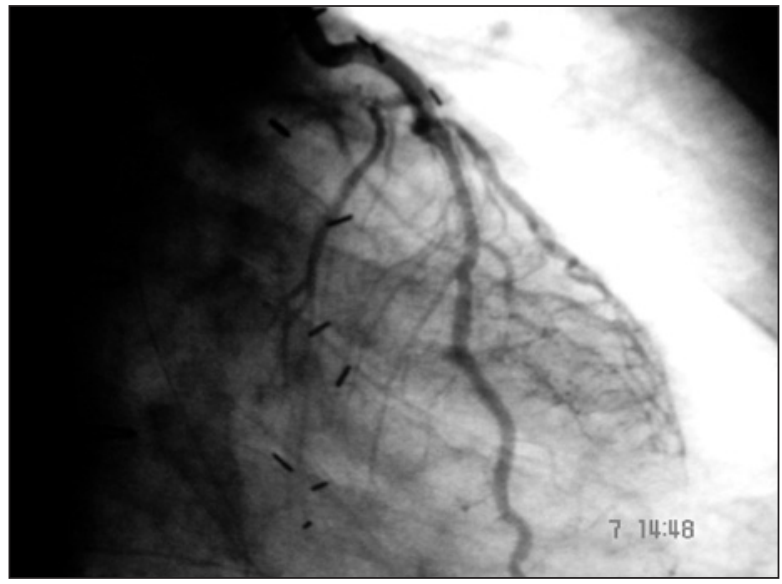

Figura 3 - Estudo da ponte da artéria torácica interna esquerda em projeção PA. A ponte da ATIE/ ACDA patente e com stent pérvio (na anastomose distal), livre de lesões obstrutivas.

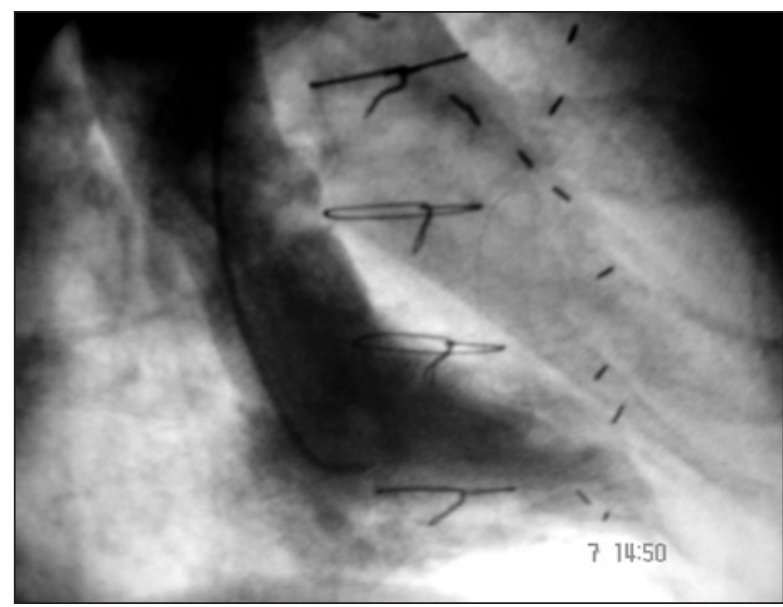

Figura 4 - Ventriculografia esquerda em projeção OAD, onde se observa acinesia ínfero-basal e demais porções com boa contratilidade. 


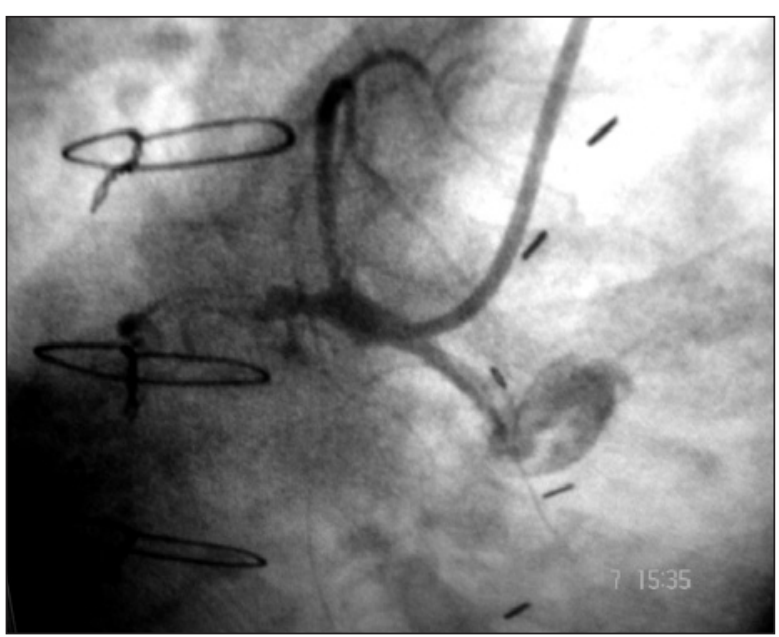

Figura 5 - Coronariografia direita em projeção OAE. Artéria coronária direita mostra-se ocluída logo após a sua origem.

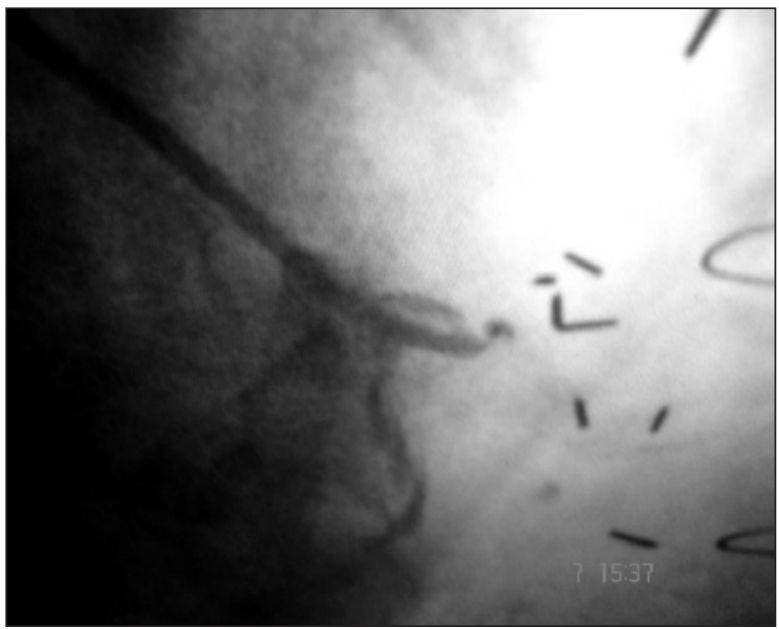

Figura 6 - Estudo da ponte de safena em projeção OAD. Observa-se outro nicho de ponte de safena.

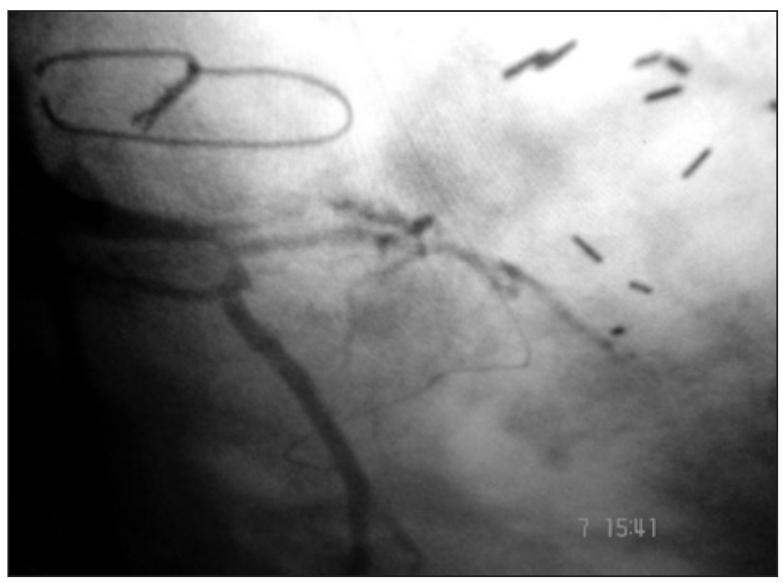

Figura 7 - Angiografia da coronária esquerda (Pré-PTCA) em projeção OAD caudal. Lesão obstrutiva grave na porção distal do tronco coronariano esquerdo.

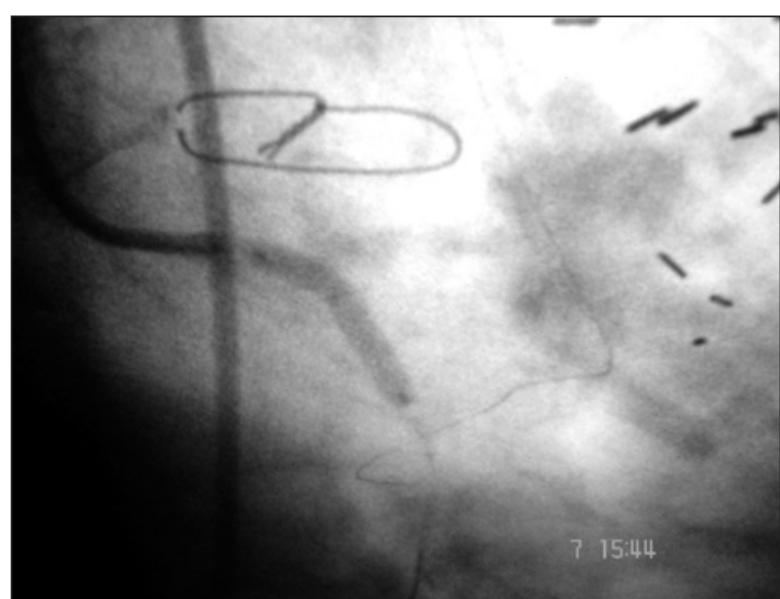

Figura 8 - PTCA de tronco coronariano esquerdo protegido/artéria circunflexa com implante de stent farmacológico. Implantado stent TAXUS - Liberté $(3,0 \times 16)$ a 16 atm, pela técnica direct stent.

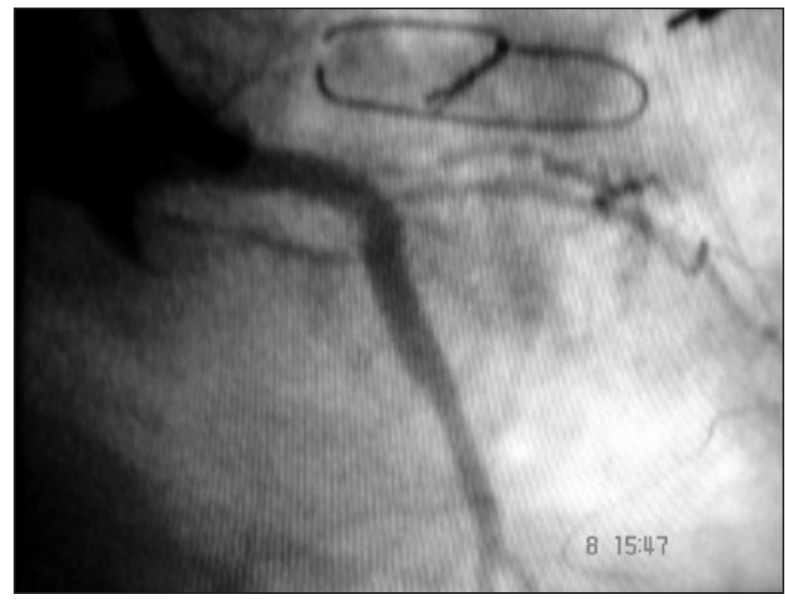

Figura 9 - PTCA de tronco coronariano esquerdo protegido/artéria cirflexa com stent com eluição de paclitaxel. Resultado angiográfico final da artéria coronária esquerda, após PTCA, em projeção OAD caudal.

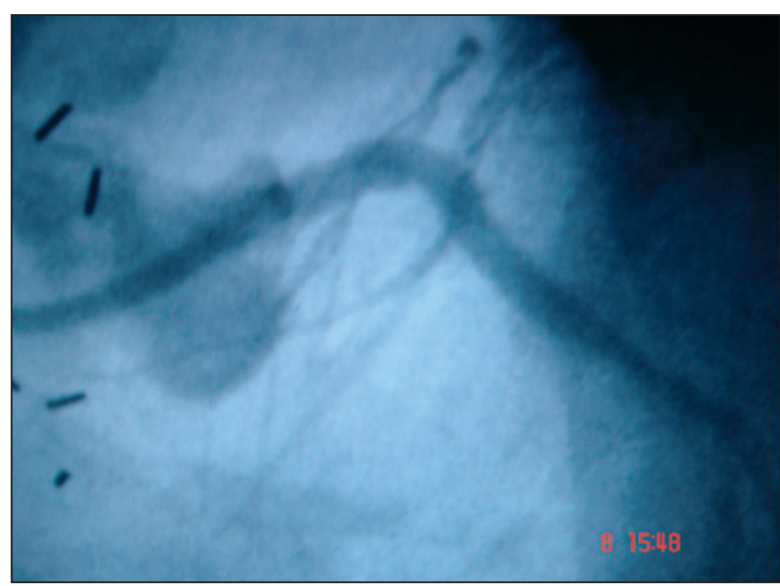

Figura 10 - PTCA de tronco coronariano esquerdo protegido/artéria circunflexa com stent paclitaxel. Angiografia da artéria coronária esquerda de controle, após PTCA, em projeção OAE caudal. 
desde 2003 e havia necrose na parede inferior. Achamos que a artéria culpada seria a artéria coronária esquerda, pois angiograficamente houve progressão da lesão do TCE/ ACX, havendo contratilidade preservada na região irrigada e sendo tecnicamente possível o tratamento percutâneo. A antiagregação plaquetária com os inibidores da glicoproteína Ilb/Ila foi necessária, pois o paciente apresentava dor precordial no momento do exame, apesar da terapêutica antisquêmica otimizada, optamos por não esperar por mais 6h (após a dose de ataque do clopidogrel) em decorrência da gravidade do quadro clínico. Após o implante da endoprótese, não houve alteração da perviedade da artéria descendente anterior, uma vez que esta já se encontrava ocluída no $1 / 3$ proximal anteriormente. Concluímos que, o tratamento percutâneo em lesões de TCE protegido, com stent com eluição de drogas antiproliferativas, é uma alternativa viável em paciente de alto risco, porém são necessários estudos com maior número de doentes e maior tempo de seguimento.

\section{REFERÊNCIAS BIBLIOGRÁFICAS}

1. Serruys PW, Unger F, Souza JE, Jatene A, Bonnier HJ, Schonberger JP, et al. Comparison of coronary-artery bypass surgery and stenting for the treatment of multivessel disease. N Engl J Med. 2001;344(15):1117-24.

2. Smith SC, Feldman TE, Hirshfeld JW, Jacobs AK, Kern MJ, King SB $3^{\text {rd }}$, et al. American College of Cardiology/American Heart Association/ Society for Cardiovascular Angiography and Interventions Writting Committee to Update the 2001
Guidelines for Percutaneous Coronary Intervention. ACC AHA/SCAI 2005 Guideline Update for Percutaneous Coronary Intervention- summary article: a report of the American College of Cardiology/American Heart Association Task Force on Practice Guidelines (ACC/AHA/SCAI Writing Committee to Update the 2001 Guidelines for Percutaneous Coronary Intervention). Circulation. 2006;113(1):156-75.

3. Chieffo A, Stankovic G, Bonizzoni E, Tsagalou E, lakovou I, Montorfano M, et al. Early and mid-term results of drugeluting stent implantation in unprotected left main. Circulation. 2005;111(6):791-5.

4. Valgimigli $M$, van Mieghem CA, Ong AT, Aoki J, Granillo GA, McFadden EP, et al. Short- and long-term clinical outcome after drug-eluting stent implantation for the percutaneous treatment of left main coronary artery disease: insights from the Rapamycin- Eluting and Taxus Stent Evaluated at Rotterdam Cardiology Hospital registries (RESEARCH and TSEARCH). Circulation. 2005;111(11):1383-9.

5. Park SJ, Kim YH, Lee BK, Lee SW, Lee CW, Hong MK, et al. Sirolimus-eluting stent implantation for unprotected left main coronary artery stenosis: comparison with bare metal stent implantation. J Am Coll Cardiol. 2005;45(3):351-6.

6. Lee MS, Kapoor N, Jamal F, Czer L, Aragon J, Forrester J, et al. Comparison of coronary artery bypass surgery with percutaneous coronary intervention with drug-eluting stents for unprotected left main coronary artery disease. J Am Coll Cardiol. 2006;47(4):864-70.

7. Baim DS, Mauri L, Cutlip DC. Drug-eluting stenting for unprotected left main coronary artery disease: are we ready to replace bypass surgery? J Am Coll Cardiol. 2006;47(4):878-81.

8. Price MJ, Cristea E, Sawhney N, Kao JA, Moses JW, Leon $M B$, et al. Serial angiographic follow-up of sirolimus-eluting stents for unprotected left main coronary artery revascularization. J Am Coll Cardiol. 2006;47(4):871-7. 